\title{
Securing Location-Aware Services Based on Online/Offline Signatures in VANETs ${ }^{\star}$
}

\author{
Chul Sur ${ }^{1}$, Youngho Park ${ }^{2}$, Takashi Nishide ${ }^{1}$, \\ Kouichi Sakurai ${ }^{1}$, and Kyung Hyune Rhee ${ }^{2, \star \star}$ \\ 1 Graduate School of Information Science and Electrical Engineering, \\ Kyushu University, Japan \\ \{chulsur, nishide, sakurai\}@itslab.csce.kyushu-u.ac.jp \\ 2 Department of IT Convergence and Application Engineering, \\ Pukyong National University, Republic of Korea \\ \{pyhoya, khrhee\}@pknu.ac.kr
}

\begin{abstract}
In this paper, we propose a novel privacy-preserving location assurance protocol for secure location-aware services over vehicular ad hoc networks (VANETs). In particular, we introduce the notion of location-aware credentials based on "hash-sign-switch" paradigm so as to guarantee the trustworthiness of location in location-aware services while providing conditional privacy preservation which is a desirable property for secure vehicular communications. Furthermore, the proposed protocol provides efficient procedures that alleviate a burden of computation for location-aware signature generation and verification on vehicles in VANETs. In order to achieve these goals, we consider online/offline signature scheme and identity-based aggregate signature scheme as our building blocks. Finally, we demonstrate experimental results to confirm the efficiency and effectiveness of the proposed protocol.
\end{abstract}

Keywords: VANETs, Location Assurance, Privacy Preservation, Location-Aware Credential, Online/Offline Signatures.

\section{Introduction}

Vehicular ad hoc networks (VANETs) have emerged as a promising research field to provide significant opportunities for the deployment of a variety of applications and services as well as intelligent transportation systems to users. A VANET mainly consists of on-board units (OBUs) and roadside units (RSUs), where OBUs are installed on vehicles to provide wireless communication capability, while RSUs are deployed to provide access point to vehicles within their radio coverage. By this organization, the VANET enables useful functions, such

\footnotetext{
* This work was supported by the National Research Foundation of Korea Grant funded by the Korean Government(Ministry of Education, Science and Technology). [NRF-2010-357-D00223].

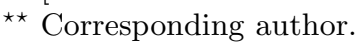


as cooperative driving and probe vehicle data, that increase vehicular safety and reduce traffic congestion, and offer access to location-aware service applications.

A location-aware service on a VANET is to provide time-sensitive and higherlevel services that distribute on-demand information such as traffic conditions, weather, and available facilities (e.g., gas station or restaurant) for a certain geographic area of interest by taking advantage of vehicular communications [4. For the sake of supporting such a useful service, Dikaiakos et al. 44 proposed the development and deployment of location-aware service infrastructure on top of emerging VANETs based on a vehicular information transfer protocol (VITP) [5] which is an application layer communication protocol specifying the syntax and the semantics of messages for a VANET service. However, VITP does not provide built-in security features although it is necessary to develop a suit of elaborate and carefully designed security mechanisms before all other implementation aspects of VANETs [17.

Upon taking security design for viable location-aware service applications into consideration, location assurance is a fundamental security requirement from user's perspective because location information is an indispensable aspect for guaranteeing a reliable and trustworthy location-aware service. Recent advances in localization technologies enable accurate location estimation of vehicles based on transmission signal properties such as signal strength and direction. Prior location verification schemes 111618] in the literature focused on secure packet forwarding in geographic routing protocol to identify a false node, which fakes its position, by verifying whether a neighbor node physically resides within a communication range. However, this approach is different from our protocol, and further, cannot support location assurance from the view of location-aware service.

On the other hand, sensitive information such as the identity and location privacy of a vehicle should be preserved from being illegally traced by a global eavesdropping attacker through vehicular communications [17. To satisfy the requirement of privacy preservation, a variety of privacy-preserving authentication protocols have been proposed on the basis of digital signature including group signature schemes and anonymous certificates using pseudonyms of vehicles to conceal the real identities of vehicles 121315 . However, those protocols cannot fulfill the location assurance requirement in our mind. Moreover, the requirement of location assurance seems to conflict with location privacy.

Our Contribution. In this paper, we propose a novel and efficient privacypreserving location assurance protocol that addresses the conflicting goals of privacy preservation and location assurance for location-aware services over VANETs. Even though ordinary digital signature schemes are sufficient to guarantee the authenticity of a message including location information, it is insufficient to guarantee the semantics that the message was responded from a vehicle that passed through the claimed location since there is no binding between the signature function and the location information. Consequently, we introduce the notion of location-aware credential which is a signature on a trapdoor hash 
value [10] under geographic location information and can be transformed into location-aware signatures on location-aware messages by applying "hash-signswitch" paradigm [20] without violating location privacy of vehicles through vehicular communications. Moreover, the proposed protocol gains merit from the performance point of view by providing efficient signature generation and even verification on vehicles. In order to achieve these goals, we elaborately incorporate online/offline signatures [3] with an identity-based aggregate signature scheme [19] to generate location-aware credentials and signatures for location assurance, and make use of pseudonym-based anonymous authentication for privacy preservation.

\section{System Model}

\subsection{Architecture}

In this section, we describe our system model, in which communication nodes are either the trusted authority (TA), RSUs, or OBUs. The detailed description of system components is as follows:

- TA is public agencies or corporations with administrative powers in a specific field; for example, city or state transportation authorities. The TA is in charge of the registration of RSUs and vehicles deployed on a VANET, and issues cryptographic quantities through initial registration. In addition, the TA should be able to trace the real identity of a message originator by law enforcement when a problematic situation occurs.

- RSUs are subordinated to the TA and responsible for issuing location-aware credentials to each vehicle within RSUs' geographic areas. They assist the TA to resolve dispute cases and may not disclose any inner information without the authorization of the TA.

- OBUs are installed on the vehicles. They communicate with other OBUs for sharing location-aware information, and with RSUs for requesting the location-aware credentials used to generate signatures for a secure locationaware service.

To define architectural model more clearly, we make the following assumptions:

- RSUs are able to establish a secure channel with the TA by the Internet or any other reliable communication links with high bandwidth.

- Vehicles are equipped with an embedded computer, a GPS receiver, a wireless network interface compliant to standards like 802.11p incorporated with dedicated short range communications (DSRC) 21].

- A number of roadside service facilities (e.g., gas stations, coffee shops, restaurants, etc) are also equipped with short-range wireless interfaces and participate in the VANET.

- The TA can inspect all RSUs at high level and maintain the compromised entities list. 
Since the main goal of this paper is to design security protocol, we do not describe the process of location-aware service transactions in detail. Instead, we assume the functionalities of the VITP [5] for our underlying location-aware service on VANETs. Multi-hop message delivery can be supported by geographic routing protocol such as GPSR [8], which forwards messages toward their geographic destination.

\subsection{Security Objectives}

Here we clarify our security objectives in order to provide secure and trustworthy location-aware services among vehicles in VANET environments. The concerns of our design are summarized as follows:

- Location Assurance. A location-aware service should guarantee the semantics that the information about a certain location of interest is related to the claimed target location. That is, it must be possible for a requesting vehicle to verify that a response message was actually originated from a vehicle within the target location area.

- Authentication. Only legitimate entities should take part in the VANETs. In addition, the origin of the messages should be authenticated to guard against the impersonation and message forgery attacks.

- Location Tracking Avoidance. The real identity and location privacy of a vehicle should be preserved from illegal tracing through a vehicular communication even though location assurance is supported.

- Traceability. The authority should be able to trace the originator of a message by revealing the real identity in case of any disputed situation such as liability investigation. That is, privacy preservation protocols in a VANET must be conditional by way of precaution against problematic situations.

\section{Proposed Protocol}

In this section, we present an efficient privacy-preserving location assurance protocol consisting of system setup, OBU and RSU registration, location-aware credential issuance, and location-aware signature generation and verification. To design the protocol, we consider identity-based authenticated key agreement scheme [2] for mutual authentication between an OBU and an RSU, and online/offline signatures [3] and identity-based aggregate signature scheme [19] for efficient location-aware signature generation and verification, respectively. Especially, the essence of our protocol is to use location-aware credentials based on "hash-sign-switch" paradigm [20] for providing reliable and trustworthy locationaware services without violating the location privacy of OBUs. Table 1 describes the notations used in the proposed protocol. 
Table 1. Notations

\begin{tabular}{|c|c|}
\hline Notation & Description \\
\hline params & public system parameters \\
\hline$s k_{i}, v k_{i}$ & signing/verification key pair of entity $i$ \\
\hline$o k_{i}, r k_{j}$ & $\begin{array}{l}\text { identity-based secret keys for } \mathrm{OBU}_{i} \text { and } \mathrm{RSU}_{j} \text {, } \\
\text { respectively }\end{array}$ \\
\hline$H K_{i}, T K_{i}$ & hash key and trapdoor key for $\mathrm{OBU}_{i}$, respectively \\
\hline$\Sigma_{i, j}$ & $\begin{array}{l}\text { location-aware credential for } \mathrm{OBU}_{i} \text { issued from } \\
\mathrm{RSU}_{j}\end{array}$ \\
\hline$H_{1}, H_{2}, H_{3}, H_{4}, H_{5}$ & cryptographic hash functions \\
\hline $\mathcal{L}_{j}$ & location information of $\mathrm{RSU}_{j}$ \\
\hline$T$ & valid time period \\
\hline$M A C_{k}$ & MAC function under the key $k$ \\
\hline$E n c_{k}, D e c_{k}$ & $\begin{array}{l}\text { symmetric encryption and decryption functions } \\
\text { under the key } k \text {, respectively }\end{array}$ \\
\hline$K D F$ & key derivation function \\
\hline
\end{tabular}

\subsection{System Setup}

The TA generates the required groups and public system parameters according to 2319 . The TA chooses a multiplicative group $\mathbb{G}$ of the prime order $p$ and bilinear map groups $\left(\mathbb{G}_{1}, \mathbb{G}_{2}\right)$ of the same prime order $q$, then random generators $g \in \mathbb{G}, P \in \mathbb{G}_{1}$. Let $e: \mathbb{G}_{1} \times \mathbb{G}_{1} \rightarrow \mathbb{G}_{2}$ be a bilinear map. The TA picks random $\gamma \in \mathbb{Z}_{p}^{*}, \alpha \in \mathbb{Z}_{q}^{*}$ as the master keys for identity-based cryptography and sets $g_{0}=g^{\gamma}, P_{0}=\alpha P$ as the corresponding public keys, respectively. The TA also chooses cryptographic hash functions which are defined as $H_{1}:\{0,1\}^{*} \rightarrow \mathbb{G}_{1}$, $H_{2}:\{0,1\}^{*} \rightarrow \mathbb{Z}_{q}^{*}$ and $H_{3}, H_{4}, H_{5}:\{0,1\}^{*} \rightarrow \mathbb{Z}_{p}^{*}$.

In addition, the TA chooses a collision resistant one-way hash function $h$ and a secure symmetric encryption algorithm Enc, then defines a key derivation function $K D F$ built on the hash function $h$. Finally, it publishes the public system parameter params $=\left\{\mathbb{G}, \mathbb{G}_{1}, \mathbb{G}_{2}, g, g_{0}, e, P, P_{0}, H_{1}, H_{2}, H_{3}, H_{4}, H_{5}\right.$, Enc, KDF $\}$.

\subsection{OBU and RSU Registration}

In our system, all OBUs and RSUs need to be registered from the TA and preloaded with their own secret quantities before joining a VANET. Fig. 1 describes the procedure of initial registration with respect to OBUs and RSUs, respectively.

If the registration entity is an $\mathrm{OBU}_{i}$, it submits its own real identity $\mathrm{ID}_{i}$ to the TA. Then the TA first checks its validity. If the identity $\mathrm{ID}_{i}$ passes the check, it derives a pseudo identity $\mathrm{PID}_{i}=E n c_{K}\left(\mathrm{ID}_{i}\right)$ under the secret key $K$ from $\mathrm{OBU}_{i} \mathrm{~s}$ real identity $\mathrm{ID}_{i}$, then computes $o k_{i}=\alpha H_{1}\left(\mathrm{PID}_{i}\right)$ as $\mathrm{OBU}_{i}$ 's identitybased secret key used for mutual authentication with RSUs. The TA transmits $\left\langle\right.$ params, $\left.\mathrm{PID}_{i}, o k_{i}\right\rangle$ to $\mathrm{OBU}_{i}$ and registers $\left\langle\mathrm{ID}_{i}, \mathrm{PID}_{i}\right\rangle$ as a legitimate entity in secure storage. 


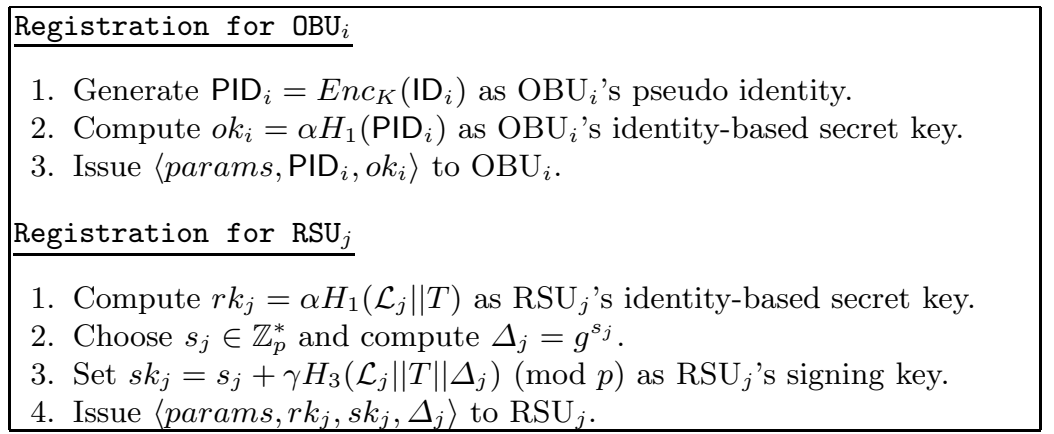

Fig. 1. Initial registration for OBUs and RSUs by the TA

On the other hand, if the registration entity is an $\mathrm{RSU}_{j}$, the TA computes $r k_{j}=\alpha H_{1}\left(\mathcal{L}_{j} \| T\right)$ and $s k_{j}=s_{j}+\gamma H_{3}\left(\mathcal{L}_{j}\|T\| \Delta_{j}\right)(\bmod p)$ using the location information $\mathcal{L}_{j}$ in which $\mathrm{RSU}_{j}$ is located together with the valid time period $T$. At this step, $r k_{j}$ is an identity-based secret key used for mutual authentication with OBUs and $s k_{j}$ is a signing key which is used for issuing location-aware credentials for assuring location-aware services in VANETs, respectively. Then the TA issues $\left\langle\right.$ params, $\left.r k_{j}, s k_{j}, \Delta_{j}\right\rangle$ to the $\mathrm{RSU}_{j}$.

Remark 1. According to 4, a location information $\mathcal{L}$ can be represented as two-value tuples [road-ID, segment-ID], where road-ID is a unique key representing a road and segment-ID is a number representing a segment of that road [14]. Given that the movement of vehicles is constrained within the road system, we can assume that the geographic areas of interest are restricted to roads and road segments. Therefore, those representations can be used as identifiers for our key generation.

Remark 2. The valid time period $T$ used in our protocol makes fine-grained revocation possible with respect to $\mathrm{RSU}_{j}$ 's identity-based secret quantities. For instance, if the TA sets the valid time period $T$ as current date to generate $r k_{j}=\alpha H_{1}\left(\mathcal{L}_{j} \| T\right)$ and $s k_{j}=s_{j}+\gamma H_{3}\left(\mathcal{L}_{j}\|T\| \Delta_{j}\right)$, the vulnerability window of $\mathrm{RSU}_{j}$ is restricted to the end of the day since $\mathrm{RSU}_{j}$ 's secret keys are inherently useless after current date. Moreover, the process of secret key renewal on the TA is insignificant operation since only hash function, 1 point multiplication of $\mathbb{G}_{1}$ and 1 modular exponentiation of $\mathbb{Z}_{p}^{*}$ are used in our protocol and pre-computations are also possible.

\subsection{Location-Aware Credential Issuance}

When an $\mathrm{OBU}_{i}$ wants to get a new location-aware credential for joining secure location-aware service from the $\mathrm{RSU}_{j}$ located in the $\mathrm{OBU}_{i}$ 's geographic area, 
the $\mathrm{OBU}_{i}$ and the $\mathrm{RSU}_{j}$ perform a location-aware credential issuance protocol. The proposed protocol is composed of two phases. One is mutual authentication between the $\mathrm{OBU}_{i}$ and the $\mathrm{RSU}_{j}$ using their identity-based secret keys, and the other is a location-aware credential generation by the $\mathrm{RSU}_{j}$. The detailed steps are as follows.

Step 1. The $\mathrm{OBU}_{i}$ picks a random $a \in \mathbb{Z}_{q}^{*}$ to compute $X=a P$ and generates $Q_{i}=H_{1}\left(\mathrm{PID}_{i}\right)$, then sends $\left\langle X, Q_{i}\right\rangle$ to the $\mathrm{RSU}_{j}$ as a request.

Step 2. Upon receiving the request, the $\mathrm{RSU}_{j}$ picks a random $b \in \mathbb{Z}_{q}^{*}$ and computes $Y=b P$. The $\mathrm{RSU}_{j}$ establishes $k=e\left(b Q_{i}, P_{0}\right) \cdot e\left(r k_{j}, X\right)$ and computes $\pi_{j}=M A C_{k_{0}}\left(Q_{i}, X, Y, \mathcal{L}_{j}, T\right)$, where $k_{0}=K D F(k \| 0)$. Then the $\mathrm{RSU}_{j}$ sends $\left\langle Y, \mathcal{L}_{j}, T, \pi_{j}\right\rangle$ to the $\mathrm{OBU}_{i}$ as a response.

Step 3. The $\mathrm{OBU}_{i}$ also establishes $k=e\left(o k_{i}, Y\right) \cdot e\left(a Q_{j}, P_{0}\right)$ and checks that $\pi_{j} \stackrel{?}{=} M A C_{k_{0}}\left(Q_{i}, X, Y, \mathcal{L}_{j}, T\right)$ to authenticate the $\operatorname{RSU}_{j}$, where $Q_{j}=$ $H_{1}\left(\mathcal{L}_{j} \| T\right)$ and $k_{0}=K D F(k \| 0)$. If it holds, the $\mathrm{OBU}_{i}$ chooses a random $x_{i} \in \mathbb{Z}_{q}^{*}$ as a trapdoor key $T K_{i}$ and sets $H K_{i}=x_{i} P$ as the corresponding hash key, respectively. Finally, the $\mathrm{OBU}_{i}$ computes $C_{i}=E n c_{k_{1}}\left(\mathrm{PID}_{i}, H K_{i}\right)$, where $k_{1}=K D F(k \| 1)$ and $\pi_{i}=M A C_{k_{0}}\left(\mathrm{PID}_{i}, Q_{i}, X, Y, H K_{i}, \mathcal{L}_{j}, T\right)$, then transmits $\left\langle C_{i}, \pi_{i}\right\rangle$ to the $\mathrm{RSU}_{j}$.

Step 4. First, the $\mathrm{RSU}_{j}$ decrypts $C_{i}$ under $k_{1}=K D F(k \| 1)$ to obtain OBU's pseudo identity $\mathrm{PID}_{i}$ and hash key $H K_{i}$. Then it looks up the up-to-date revocation list retrieved from the TA to check the validity of the given $\mathrm{PID}_{i}$. If the $\mathrm{PID}_{i}$ is revoked one, the $\mathrm{RSU}_{j}$ refuses to issue a location-aware credential. Otherwise, it checks that $\pi_{i} \stackrel{?}{=} M A C_{k_{0}}\left(\mathrm{PID}_{i}, Q_{i}, X, Y, H K_{i}, \mathcal{L}_{j}, T\right)$. If the check holds, the $\mathrm{RSU}_{j}$ chooses a random $\lambda_{i} \in \mathbb{Z}_{q}^{*}$ and computes the trapdoor hash value $\xi_{i}=\lambda_{i} H K_{i}$. The $\mathrm{RSU}_{j}$ also picks a random $r \in \mathbb{Z}_{p}^{*}$, then generates a location-aware credential $\Sigma_{i, j}=\left(\Delta_{j}, U_{i}, V_{i}\right)$ :

$$
\left\{\begin{array}{l}
U_{i}=g^{r} \\
V_{i}=r \psi_{i, 0}+s k_{j} \psi_{i, 1}(\bmod p)
\end{array}\right.
$$

where $\psi_{i, 0}=H_{4}\left(\xi_{i}\left\|\mathcal{L}_{j}\right\| T\left\|U_{i}\right\| \Delta_{j}\right)$ and $\psi_{i, 1}=H_{5}\left(\xi_{i}\left\|\mathcal{L}_{j}\right\| T\left\|\psi_{i, 0}\right\| U_{i} \| \Delta_{j}\right)$. Finally, the $\mathrm{RSU}_{j}$ computes $C_{j}=\operatorname{Enc}_{k_{1}}\left(\lambda_{i}\right)$ and $\pi_{j}^{\prime}=M A C_{k_{0}}\left(\lambda_{i}, \Sigma_{i, j}\right)$, then transmits $\left\langle C_{j}, \Sigma_{i, j}, \pi_{j}^{\prime}\right\rangle$ to the $\mathrm{OBU}_{i}$. In addition, $\mathrm{RSU}_{j}$ stores $\left\langle\mathrm{PID}_{i}, H K_{i}\right\rangle$ in its local credential list for assisting the TA by way of provision against a liability investigation. Note that, in location-aware credential generation, no identity-related information is included in $\Sigma_{i, j}$.

Step 5. The $\mathrm{OBU}_{i}$ retrieves the secret value $\lambda_{i}=D e c_{k_{1}}\left(C_{j}\right)$, then checks $\pi_{j}^{\prime} \stackrel{?}{=} M A C_{k_{0}}\left(\lambda_{i}, \Sigma_{i, j}\right)$. If the check is valid, the $\mathrm{OBU}_{i}$ sets $s k_{i}=\left\langle T K_{i}, \lambda_{i}\right\rangle$ as its singing key and $v k_{i}=H K_{i}$ as its verification key, respectively.

Remark 3. The location-aware credential $\Sigma_{i, j}=\left(\Delta_{j}, U_{i}, V_{i}\right)$ for $\mathrm{OBU}_{i}$ is an identity-based signature on the trapdoor hash value $\xi_{i}$ under the geographic 
location $\mathcal{L}_{j}$ and the valid time period $T$. Moreover, the location-aware credential $\Sigma_{i, j}$ can be re-used whenever $\mathrm{OBU}_{i}$ wants to sign a location-aware message during the specific time period $T$.

\subsection{Location-Aware Signature Generation and Verification}

Fig. 2 depicts the message structure used for a secure location-aware service. The type field represents either request or response. The target and source fields contain location area of interest that specifies the road and segment identifiers, as retrieved by an on-board navigation and positioning system. Hash_key and signature fields contain message originator's hash key and a digital signature on the message under location information and valid time period, respectively.

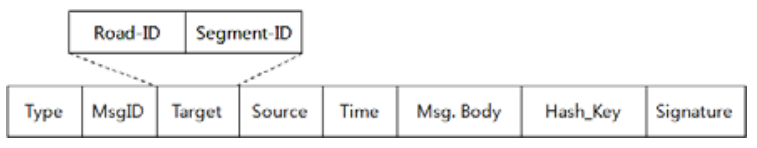

Fig. 2. Message structure

As aforementioned, since we assume an underlying VANET routing protocol, we just present how location-aware credential lead to location-aware signature generation and verification on the message for a secure and trustworthy locationaware service as shown in Fig. 3. For a given location-aware service message $m$, any entity in a VANET is able to convert a location-aware credential $\Sigma$ into a location-aware signature $\sigma$ on the message $m$ by using its own trapdoor key $T K$ during the valid time period $T$.

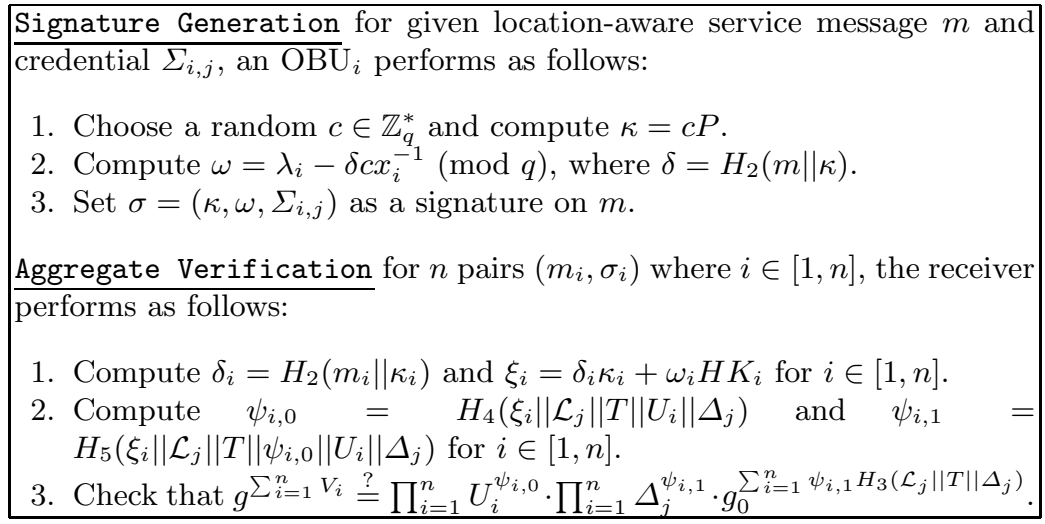

Fig. 3. Location-aware signature generation and verification 
The consistency of the location-aware signature verification can be proved as follows:

- For the trapdoor hash value $\xi_{i}$, we have that

$$
\begin{aligned}
\xi_{i} & =\delta_{i} \kappa_{i}+\omega_{i} H K_{i} \\
& =H_{2}\left(m_{i} \| \kappa_{i}\right) \kappa_{i}+\omega_{i} H K_{i} \\
& =H_{2}\left(m_{i} \| c_{i} P\right) c_{i} P+\left(\lambda_{i}-H_{2}\left(m_{i} \| c_{i} P\right) c_{i} x_{i}^{-1}\right) \cdot x_{i} P \\
& =\lambda_{i} H K_{i}
\end{aligned}
$$

- For the verification check, we have that

$$
\begin{aligned}
g^{\sum_{i=1}^{n} V_{i}} & =g^{\sum_{i=1}^{n} r_{i} \psi_{i, 0}+\sum_{i=1}^{n} s k_{j} \psi_{i, 1}} \\
& =g^{\sum_{i=1}^{n} r_{i} \psi_{i, 0}} \cdot g^{\sum_{i=1}^{n} s k_{j} \psi_{i, 1}} \\
& =\prod_{i=1}^{n}\left(g^{r_{i}}\right)^{\psi_{i, 0}} \cdot g^{\sum_{i=1}^{n}\left(s_{j}+\gamma H_{3}\left(\mathcal{L}_{j}\|T\| \Delta_{j}\right)\right) \psi_{i, 1}} \\
& =\prod_{i=1}^{n} U_{i}^{\psi_{i, 0}} \cdot \prod_{i=1}^{n} \Delta_{j}^{\psi_{i, 1}} \cdot g_{0}^{\sum_{i=1}^{n} \psi_{i, 1} H_{3}\left(\mathcal{L}_{j}\|T\| \Delta_{j}\right)}
\end{aligned}
$$

The proposed protocol is highly efficient in terms of signature generation and even verification since only 1 point multiplication of $\mathbb{G}_{1}$, and 2 point multiplications of $\mathbb{G}_{1}$ and 4 modular exponentiations of $\mathbb{Z}_{p}^{*}$ are required in signature generation and verification phases, respectively. Moreover, for $n$ messages with signatures $\sigma_{i}(1 \leq i \leq n)$ replied from $n$ vehicles, the receiver aggregately verifies the $n$ signatures to significantly reduce the computational costs.

\section{Security Analysis}

In this section, we analyze how the proposed protocol satisfies the security objectives stated in Section 2.2.

- Location Assurance. This goal can be satisfied by the location-aware credential $\Sigma$ which is a signature under a location information $\mathcal{L}$ and a time period $T$. If the location-aware credential in a location-aware signature $\sigma$ is verified as valid by using the location information $\mathcal{L}$ and the time period $T$ specified in a location-aware message, then the verifier can be convinced that the message was responded by an OBU that passed through the claimed location $\mathcal{L}$ for given time period $T$ because the location-aware credential for the OBU is issued by the RSU physically located in the target geographic area. Moreover, since the location-aware credential is generated by an identity-based aggregate signature scheme [19] which was proven to be secure against adaptive chosen message attacks, no adversary can launch a forgery attack against the location-aware credential. 
- Authentication. The authenticity of entities that participated in a VANET can be assured by the identity-based secret keys issued through the initial registration in the protocol. That is, only the RSU possessing a valid $r k$ corresponding to its location and the OBU possessing a valid $o k$ derived from its pseudo identity can be authenticated to each other. Therefore, when we assume the security of the underlying identity-based cryptography, no one can launch an impersonation attack unless the entity is registered to the TA. To forge location-aware signatures based on online/offline signatures [3], and further, an adversary should find collisions of the trapdoor hash value $\xi$ given the corresponding hash key $H K$. However, this implies the adversary can solve the discrete logarithm problem in $\mathbb{G}_{1}$, which is computationally infeasible.

- Location Tracking Avoidance. In our protocol, message senders and receivers are specified by their hash keys. The distribution of hash key $H K$ is computationally indistinguishable from uniform distribution in $\mathbb{G}_{1}$ if the probability over the choice of $x$ is uniformly distributed in $\mathbb{Z}_{q}^{*}$. Therefore, indistinguishability of hash keys can prevent an adversary from identifying OBUs. In addition, since the hash key is renewed whenever an OBU enters into different geographic areas, an attacker cannot match the originators between observed messages from different locations. As a result, unlinkability of hash keys at different locations can prevent a global eavesdropper from tracking movement of an OBU.

- Traceability. In dispute case, the TA is involved in tracing the originator of the message. Given a message formed as shown in Fig. 2, the TA first retrieves the location information $\mathcal{L}_{j}$ and originator's hash key $H K_{i}$ from the message. Then the TA requests the pseudo identity $\mathrm{PID}_{i}$ corresponding to the hash key $H K_{i}$ to the $\mathrm{RSU}_{j}$ located in $\mathcal{L}_{j}$. On TA's demand, the $\mathrm{RSU}_{j}$ searches the $\mathrm{PID}_{i}$ from its local credential list and responds with the $\mathrm{PID}_{i}$. Finally, the TA can recover the real identity $\mathrm{ID}_{i}$ by decrypting the $\mathrm{PID}_{i}$ under TA's secret key $K$.

\section{Performance Evaluation}

In this section, we evaluate the performance of the proposed protocol in terms of RSU location-aware credential issuance, message processing rate of a responding vehicle, and message processing delay for reply messages. In order to evaluate the processing time of location-aware credential issuance protocol, and locationaware signature generation and verification, we considered PBC library [23] for implementing bilinear pairing and modular operations with 1024 bits security level on Pentium IV $3 \mathrm{GHz}$.

Table 2 shows the measures to estimate the proposed protocol. Since the computations of a bilinear pairing $t_{p}$, a point multiplication $t_{m}$ and a modular exponentiation $t_{e}$ are much time consuming operations, we did not account any other negligible computation such as cryptographic hash functions. 
Table 2. The number of cryptographic operations and the processing time

\begin{tabular}{c||c|c||c}
\hline & RSU & OBU & Time $(\mathrm{ms})$ \\
\hline \hline Credential issuance & $2 t_{p}+3 t_{m}+1 t_{e}$ & $2 t_{p}+3 t_{m}$ & 37.3 \\
\hline Msg. signing & - & $1 t_{m}$ & 1.9 \\
\hline Msg. verifying & - & $2 t_{m}+4 t_{e}$ & 12.2 \\
\hline
\end{tabular}

\subsection{Processing of RSU Location-Aware Credential Issuance}

The main operation of an RSU is to issue location-aware credentials to vehicles on requests within RSUs valid coverage range $R_{r n g}$. Hence, RSU's performance always depends on vehicles density $d$ and speed $v$ within the coverage range. The RSU valid serving ratio $S_{R S U}$, which is the fraction of the number of actually issued credentials to the number of requests [13], can be defined by

$$
S_{R S U}= \begin{cases}1, & \text { if } \frac{R_{r n g}}{T_{k} \cdot v} \cdot \frac{1}{d \cdot \rho} \geq 1 \\ \frac{R_{r n g}}{T_{k} \cdot v} \cdot \frac{1}{d \cdot \rho}, & \text { otherwise. }\end{cases}
$$

where $\rho$ is the probability for each vehicle to request a location-aware credential and $T_{k}$ is the execution time of location-aware credential issuance protocol.

Fig. 4 depicts the RSU valid serving ratio with different vehicle density and different vehicle speed for $R_{r n g}=500 \mathrm{~m}$, and the probability $\rho=0.8$ and $\rho=1.0$, respectively. From the results, we can observe that RSU can sufficiently deal with the location-aware credential requests in most practical scenarios even though RSU cannot fully process credential issuance protocol if more than 320 vehicles request their location-aware credentials with the probability greater than 0.8 at the same time. Thus, we conclude that the proposed location-aware credential issuance protocol is feasible.

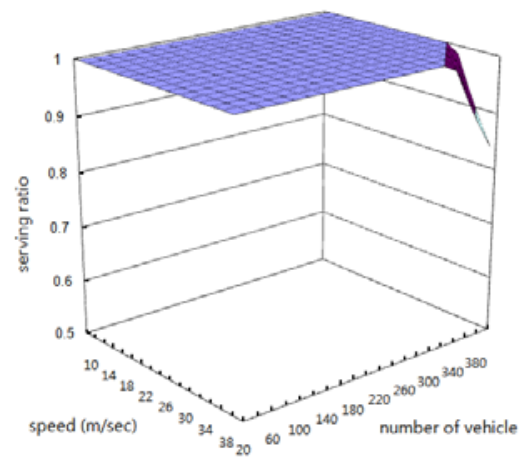

(a) valid serving ratio for $\rho=0.8$

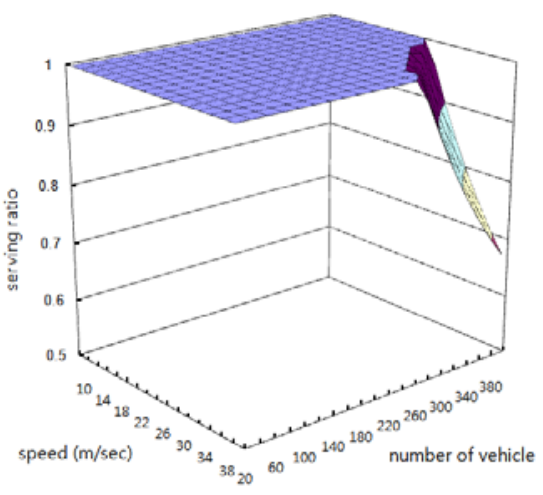

(b) valid serving ratio for $\rho=1.0$

Fig. 4. RSU valid serving ratio for location-aware credential issuance 


\subsection{Processing of Responding Vehicle}

Within a target location area, the valid response processing ratio of a responding vehicle is estimated as the fraction of the actually processed location-aware responses to the number of received requests while the responding vehicle goes through the area after obtaining a location-aware credential. A serving duration $T_{D}$ for the vehicle passing the target area can be computed as $T_{D}=$ $\left(R_{r n g} / v\right)-T_{k}$. Let $T_{s}$ and $T_{v}$ be the processing times for signature generation and verification, respectively. Since the responding vehicle requires a signature verification for a request message and a signature generation for a response message, the number of response $N_{\text {res }}$ which the responding vehicle can deal with is measured by $N_{\text {res }}=T_{D} /\left(T_{s}+T_{v}\right)$. Let $N_{r}$ be a request message rate per second, and $V_{n}$ be the average number of requesting vehicle for a target area. Then, the number of request $N_{r e q}$ received while passing the target area is $N_{r e q}=\left(R_{r n g} / v\right) \cdot N_{r} \cdot V_{n}$, and then the response ratio $S_{r e s}$ can be evaluated as $N_{\text {res }} / N_{\text {req }}$.

$$
S_{r e s}= \begin{cases}1, & \text { if } \frac{T_{D} /\left(T_{s}+T_{v}\right)}{\left(R_{r n g} / v\right) \cdot N_{r} \cdot V_{n}} \geq 1 ; \\ \frac{T_{D} /\left(T_{s}+T_{v}\right)}{\left(R_{r n g} / v\right) \cdot N_{r} \cdot V_{n}}, & \text { otherwise. }\end{cases}
$$

Fig. 5 depicts serving ratio of a responding vehicle with $20 \mathrm{~m} / \mathrm{s}$ speed in $300 \mathrm{~m}$ segment area depending on the number of requesting vehicles and message rates. From the results, if a message rate is higher than two messages per second and more than 32 vehicles send request messages, the responding vehicle cannot fully process the all requests. However, location-aware service is an on-demand service and the VITP puts a longer time interval than one second as considering the replying phase processing delay. Consequently, the proposed protocol can practically process almost all location-aware requests in a secure manner.

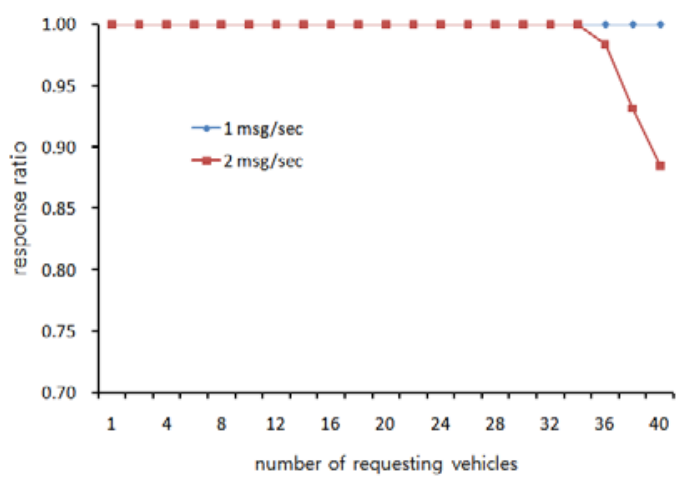

Fig. 5. Serving ratio of a responding vehicle in a target area depending on query message rate 


\subsection{Simulation Result}

To evaluate the performance for the proposed secure location-aware message query and response over VANETs, we simulated vehicular communications considering highway-traffic scenario by using NS-2 simulator. We used the GPSR [8] as a geographic routing protocol provided by 9 and IEEE $802.11 \mathrm{p}$ wireless interface configuration [22] with $11 \mathrm{Mbps}$ bandwidth and $250 \mathrm{~m}$ nominal transmission range.

In our highway-traffic scenario, we deployed vehicles on 5km-long road with 3 lanes to each direction, and fixed target road segment range to $300 \mathrm{~m}$. Then, we estimated the message processing delay by varying the query distance to the target area and inter-vehicle space on the road, respectively.

The left part in Fig. 6 shows the message processing delays to the query distance from $500 \mathrm{~m}$ to $2,500 \mathrm{~m}$ and $n$ response messages within $300 \mathrm{~m}$ target road segment, where $n=5,10,15$. The delay was measured by end-to-end round-trip time and location-aware signature generation and verification time. However, we did not take into account message loss suffered from routing failure. In addition, the right part in Fig. 6 shows the message processing delays depending on a vehicle density to $2,000 \mathrm{~m}$ query distance. To measure the processing delay, we varied the inter-vehicle space from $50 \mathrm{~m}$ to $150 \mathrm{~m}$, respectively. From the result, we can observe that the longer inter-vehicle space, which means sparse density, increases the message transmission delay due to much routing processing time.

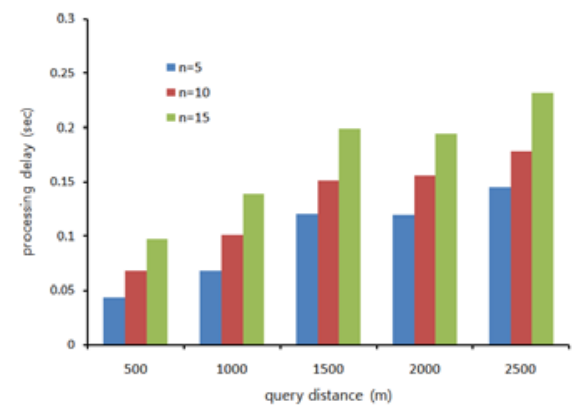

(a) delay to the query distance

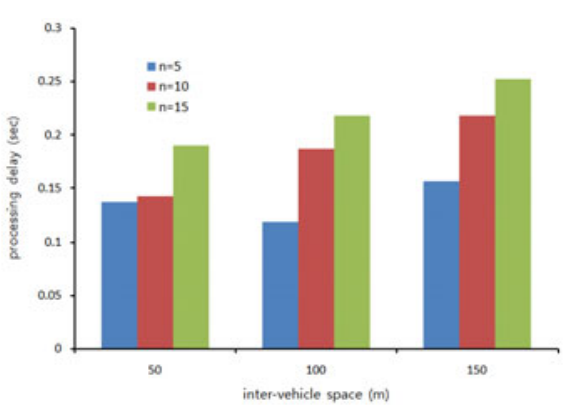

(b) delay to the inter-vehicle space

Fig. 6. Message processing delay depending on the query distance and the inter-vehicle space with the number of response messages

\section{Conclusion}

In this paper, we have proposed a novel and efficient privacy-preserving location assurance protocol for providing reliable and trustworthy location-aware services as well as privacy preservation in VANETs. In particular, we have introduced the notion of location-aware credential based on online/offline signatures and "hash-sign-switch" paradigm to guarantee the trustworthiness of location 
without violating location privacy. Furthermore, the proposed protocol provides efficient procedures for location-aware signature generation and verification to effectively alleviate computational costs on vehicles in VANETs. We have provided comprehensive analysis to confirm the fulfillment of the security objectives, and the efficiency and effectiveness of the proposed protocol.

\section{References}

1. Bellare, M., Rogaway, P.: Random oracles are practical: A paradigm for designing efficient protocols. In: ACM CCS 1993, pp. 62-73 (1993)

2. Chen, L., Chen, Z., Smart, N.P.: Identity-based key agreement protocols from pairings. International Journal of Information Security 6(4), 213-241 (2007)

3. Chen, X., Zhang, F., Susilo, W., Mu, Y.: Efficient generic on-line/off-line sinatures without key exposure. In: Katz, J., Yung, M. (eds.) ACNS 2007. LNCS, vol. 4521, pp. 18-30. Springer, Heidelberg (2007)

4. Dikaiakos, M.D., Florides, A., Nadeem, T., Iftode, L.: Location-aware services over vehicular ad-hoc networks using car-to-car communication. IEEE Journal on Selected Areas in Communications 25(8), 1590-1602 (2007)

5. Dikaiakos, M.D., Iqbal, S., Nadeem, T., Iftode, L.: VITP: An information transfer protocol for vehicular computing. In: 2nd ACM Internationial Workshop on Vehicular Ad Hoc Networks (VANET 2005), pp. 30-39 (2005)

6. Galindo, D., Garcia, F.D.: A Schnorr-like lightweight identity-based signature scheme. In: Preneel, B. (ed.) AFRICACRYPT 2009. LNCS, vol. 5580, pp. 135-148. Springer, Heidelberg (2009)

7. Gentry, C., Ramzan, Z.: Identity-based aggregate signature. In: Yung, M., Dodis, Y., Kiayias, A., Malkin, T. (eds.) PKC 2006. LNCS, vol. 3958, pp. 257-273. Springer, Heidelberg (2006)

8. Karp, B., Kung, H.: Greedy perimeter stateless routing for wireless networks. In: 6th Annual ACM/IEEE International Conference on Mobile Computing and Networking (MobiCom 2000), pp. 243-254 (2000)

9. Kiess, W., Füßler, H., Widmer, J., Mauve, M.: Hierarchical location service for mobile ad-hoc networks. ACM Sigmobile Mobile Computing and Communications Review 8(4), 47-58 (2004)

10. Krawczyk, H., Rabin, T.: Chameleon signatures. In: Symposium on Network and Distributed Systems Security (NDSS 2000), pp. 143-154 (2000)

11. Leinmuller, T., Schoch, E., Kargl, F.: Position verification approaches for vehicular ad hoc networks. IEEE Wireless Communications 13(5), 16-21 (2006)

12. Lin, X., Sun, X., Shen, X.: GSIS: A secure and privacy preserving protocol for vehicular communications. IEEE Transactions on Vehicular Technology 56(6), 3442-3456 (2007)

13. Lu, R., Lin, X., Zhu, H., Ho, P.H., Shen, X.: ECPP: Efficient conditional privacy preservation protocol for secure vehicular communications. In: IEEE INFOCOM 2008, pp. 1229-1237 (2008)

14. Nadeem, T., Dashtinezhadd, S., Liao, C., Iftode, L.: Trafficview: Traffic data dissemination using car-to-car communication. ACM Sigmobile Mobile Computing and Communications Review 8(3), 6-19 (2004)

15. Park, Y., Sur, C., Jung, C., Rhee, K.H.: An efficient anonymous authentication protocol for secure vehicular communications. Journal of Information Science and Engineering 26(3), 785-800 (2010) 
16. Pathak, V., Yao, D., Iftode, L.: Securing location aware services over VANET using geographical secure path routing. In: IEEE International Conference on Vehicular Electronics and Safety (ICVES), pp. 346-353 (2008)

17. Raya, M., Hubaux, J.-P.: Securing vehicular ad hoc networks. Journal of Computer Security 15(1), 39-68 (2007)

18. Ren, Z., Li, W., Yang, Q.: Location verification for VANETs routing. In: IEEE International Conference on Wireless and Mobile Computing, Networking and Communications, pp. 141-146 (2009)

19. Selvi, S.S.D., Vivek, S.S., Shriram, J., Rangan, C.P.: Efficient and provably secure identity based aggregate signature schemes with partial and full aggregation. Cryptography ePrint Archive, Report 2010/461 (2010)

20. Shamir, A., Tauman, Y.: Improved online/offline signature schemes. In: Kilian, J. (ed.) CRYPTO 2001. LNCS, vol. 2139, pp. 355-367. Springer, Heidelberg (2001)

21. Dedicated Short Range Communications (DSRC), http://www . leearmstrong.com/dsrc/dsrchomeset.htm

22. Overhaul of IEEE 802.11 Modeling and Simulation in NS-2, http://dsn.tm.uni-karlsruhe.de/Overhaul_NS-2.php

23. Pairing-Based Cryptography Library, http://crypto.stanford.edu/pbc

24. Simulation of Urban Mobility, http://sourceforge.net/projects/sumo 